\title{
Limited Color Display for Compressed Video
}

\author{
Ching-Min Cheng, Soo-Chang Pei and Lung-Feng Ho
}

Department of Electrical Engineering, National Taiwan University

T aipei, Tiwan, R. O.C. Email:pei@cc.ee.ntu.edu.tw

\begin{abstract} w orld wide veb (WWW) databases are in compressed formats. Therefore, it becomes an important issue to retriev e a suitable color palette from compressed domain in order to have fast and faithful color reproduction for these devices. In this paper, the color palette design methods for compressed videos are presen ted. The proposed approaches use the reduced image rather than the whole image for the color palette design to avoid the heavy computation in video decompression. Experimental results show that output image qualit yof proposed methods is acceptable to $h$ uman eyes. In addition, empirical results show that the proposed shifting-window scheme can reduce the main problem of displaying quantized image sequences, screen flicker.
\end{abstract}

Many display devices now ada ys still allo w a limitedproduce a different color palette for eac h image. Benumber of colors, called color palette, to be display ed sides, they suggest the approach of color palette filling simultaneously. Besides, images and videos in most to prev en frequent switc hingof color palettes, which

\section{Introduction}

With the prev alence of multimedia and internet, more and more digital images and videos are av ailable for people to access. The digital image or video format is usually quantized with integer from 0 to 255 for eac $\mathrm{h}$ of three color componerts (e.g. red, green, blue). All possible combinations of three of these values gives $256^{3}$ (or 16 million) distinct colors for full-color digital display. How ever,due to the costs of high speed video RAM, many current PCs and workstations generally have a single 8-bit frame buffer to allow only a limited number of colors, called color palette, to be simultaneously display ed. If an acceptable output image qualit yis desired, it is necessary to develop a useful procedure, called color quantization, for designing the color palette.

In the past, the color palette design focused on uncompressed data. For one single image, several color quantization algorithms have been proposed. Heckbert has proposed a median cut (MC) algorithm[1] where the color space is recursively divided into $M$ rectangular regions with equal color occurrence and their centroids being representation colors. Recently, the popular vector quantization ( $\mathrm{V} Q$ ) technique 4 ] is applied to the color palette design, too. The colors in input image are used as training vectors in order to refine the initial rough palette. But VQ-type algorithms are usually computational intensive such that they are not suitable for real-time processing. $P$ ei and Cheng have suggested a dependent scalar quantization (DSQ) algorithm[2], which exploits dependency of input colors and sequentially partitions the color space. The experimental results show that the DSQ can reduce the computation complexity and its output image quality is ac-

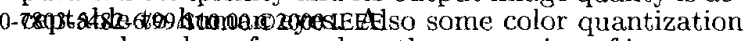
researc hers have focused on the processing of image sequences. Roytman and Gotsman[8] have proposed anII-28Besides, if only one fixed color palette is used, the
How ever,with the consideration of communication bandwidth and storage space, most image or video data now ada ysomes in compressed format. Extra hea vy computation of image or video decompression is required before any above mentioned color quantization is employed. How to design color palette directly from compressed data has thus become a significant issue. In this paper, w extend the DSQ to present some novel color palette design methods for compressed videos. The proposed methods use the reduced image in compressed domain to design color palette. w e propose a shifting-window scheme to display longer sequences without screen In section II, w edescribe the proposed approach for compressed videos, which uss the reduced image rather than the whole image for the color palette design to a void the heavy computation. In addition, the technique of extracting $k$ ey frames for compressed videos is presented and a shift-window scheme is proposed to solve the screen flicker problem. Section III reports the simulation results of proposed methods and some discussions are made.

\section{Limited Color Display for Compressed Video}

Now more and more video clips are able to be accessed in some World Wide Web databases. However, with the limit of bandwidth of in ternet, those video clips are usually compressed by using MPEG [3] format. In this condition, how to analyze the video clips to design a suitable color palette for those machines with limited color display becomes important. In this section, we will introduce methods of color palette design for MPEG compressed video.

The low-resolution DC images of MPEG video, called DC sequence, are first extracted for color palette design. Additionally, to avoid huge computation costs, key frames, which contain the major color information of the entire DC sequence, for obtaining the color palette of MPEG video. F rom the observation of DC sequences, w enoticed that color information inside a frame is unchanged for most of the sequence except key frames, which consists of color changes in the sequence. Thus, detection of color changes is essential for finding key frames. The detection of key frames is based on two steps and introduced in the following subsection:

1. detection of potential key frames

2. detection of key frames and extraction of color palette w e will only apply the DSQ to those DC images called 
degradation would get worse and worse as the sequence length gets longer. To ov ercome this problem, a multiple color palette scheme called shifting-window scheme is proposed to display compressed video with good quality even when the sequence length is getting longer.

\section{A. Extraction of DC sequence}

We will use MPEG-1 video as the example to illustrate the process of $D C$ sequence extraction. In MPEG-1, the DC coefficient of the DCT bloc $k$ in frame is the average of pixels in the bloc $k$. The DC image for I frame can be easily formed by getting the DC coefficient in every block. How ev er, the c hallenge exists in extracting $D C$ images from $P$ or $B$ frames, which use motion compensation to exploit the temporal redundancy. A general case for $\mathrm{P}$ frambas been proposed by Yeo and Liu[7] and is show ed in Figure 1 .

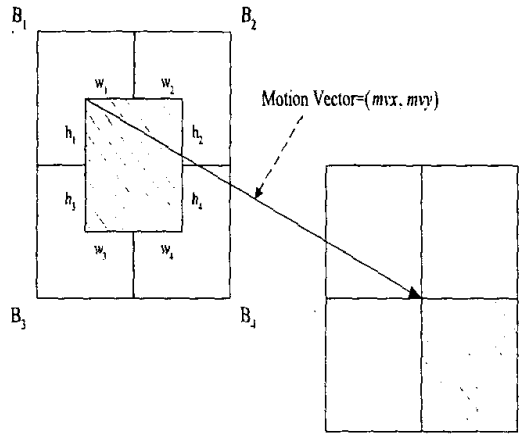

Current Block

Figure 1: The extraction of DC image.

Here, $B_{\text {ref }}$ is the block with motion vector $(m v x, m v y)$ pointing to the current bloc $\mathrm{k}$ of in terest and $B_{1}, B_{2}, B_{3}$ and $B_{4}$ are the four neighboring blocks which derive the reference block $B_{\text {ref }}$. We can express the DC component of DCT coefficients of $B_{r e f}$ denoted as $\operatorname{DCT}\left(B_{r e f}\right)$ in the following equation.

$$
\left(\operatorname{DCT}\left(B_{\text {ref }}\right)\right)_{00}=\sum_{i=1}^{4} \sum_{m=0}^{7} \sum_{l=0}^{7} w_{m l}^{i}\left(\operatorname{DCT}\left(B_{i}\right)\right)_{m l}
$$

where $w_{m l}^{i}$ are weigh ting factors related to the motion vector.

This precise calculation of DCT coefficients is timeconsuming. Since we are only interested in the DC component, the first-order approximation is used rather than precise calculation.

$$
\left(\operatorname{DCT}\left(B_{r e f}\right)\right)_{00}=\sum_{i=1}^{4} \frac{w_{i} h_{i}}{64}\left(\operatorname{DCT}\left(B_{i}\right)\right)_{00}
$$

where $w_{i}$ and $h_{i}$ are the overlapping width and height of $B_{\text {ref }}$ in block $B_{i}$. It can be sho wn that $\frac{w_{i} h_{i}}{64}$ corresponds to $w_{00}^{i}$ in Eq. 1 and this is why it's called first-order approximation. Although the approximation error will accumulate, the error is acceptable in most cases because the GOP size is usually small and the error will reset to zero at every I frame. The other advantage of this approximation is that it requires only the motion vector information and the DC values in the reference frames. This approximation approach can also be applied to $\mathrm{B}$ frame where tw o reference frames might be needed. The problem of half-pixel-wise prediction can be solv ed by using the average of largerII- bloc ks depending on the motion rector. The $9 \times 9$ block is needed if half-pixel-wise prediction occurs in both $x$ and $y$ direction. The $8 \times 9$ bloc $\mathrm{kis}$ used for half-pixel accuracy only in $x$ direction and $9 \times 8$ block is for $y$ direction only.

\section{B. Key F rame Detection for Com- pressed Video}

After the DC sequence is deriv edfrom a MPEG video, w euse its DC images for key frame detection. $T$ orepresent color information of a frame, hue component of HSV space is adopted in this paper. Hue component has been widely accepted as a good candidate of representing color difference. Using this represen tation, w ecompare the normalized difference of hue histogram betw een consecutie frames in order to detect boundaries betw een consecutive color changes. The principle behind this is that tw oframes having a unchanging background and objects will show little difference in their corresponding hue histograms. The normalization procedure is utilized for reducing the impact of noise imposing on the hue histograms of consecutive frames. The normalized hue difference betw een the hue histograms of the $l$ th and $(l-1)$ th frame, $S_{l}$ is given by the following equations:

$$
S_{l}=\sum_{j=1}^{L} N H D_{l, l-1}(j)
$$

where

$N H D_{l, l-1}(j)= \begin{cases}H_{l}^{3}(j) & \text { for } H_{l-1}(j)=0 \\ \left|\frac{H_{l}(j)-H_{l-1}(j)}{\min \left(H_{l}(j), H_{l-1}(j)\right)+1}\right| & \text { otherwise }\end{cases}$

with $H_{l}(j)$ and $H_{l-1}(j)$ being hue histograms of the tw o consecutive frames respectively and $L$ of Eq. 3 being the number of hue component bins in comparison. In Eq. 4, we choose the minimum value of $H_{l}(j)$ and $H_{l-1}(j)$ to normalize the hue difference. The condition, $H_{l-1}(j)=0$, is set to reflect the situation when pixels with the certain hue value $j$ exists in frame $l$, but not in frame $l-1$.

Similar to luminance change of the DC sequence, we ha ve obsered that color c hangeis also a local activity which inv olv es details regarding seval neighboring frames. For scene change analysis, Yeo and Liu [5] has suggested to set the threshold of luminance change in order to match the local activit $y$. We adopt this approach to detect color changes in this paper and choose a sliding window thresholding technique proposed by $Y$ eo and Liu [\$ to avoid false alarms which might occur in camera operations or object changes. In this technique, $2 n-1$ frames with $2 n-2$ h ue differences are examined in a local range. Inside this local window, a color change from $(l-1)$ th to $l$ th image occurs if the following conditions are satisfied:

1. The difference is the maximum with the window i.e., $S_{j} \leq S_{l}, j=l-n+1, \cdots, l-1, l+1, \cdots, l+n-1$.

2. $S_{l}$ is also $m$ times of the second maximum in this window.

After examination of each window, the window is shifted one frame to prepare the next examination until the whole sequence is processed. In criterion 1 , the parameter $n$ is set to be smaller than the minimum duration betw een t w o colomanges, but large enough to avoid false alarms. This is because as the window size gets smaller. the threshold is closer to be a global ap386 ach which is unfavorable for color dhange detection. 
If we set $n=30$ for a 30 frame/sec video sequence, it means that there cannot be tw o color hanges within a second. The parameter $m$ in criterion 2 is imposed to guard against some camera operations such as fast panning or zooming. F or these operations, the hue differences $S_{l}$ w ould maintain consecutive peaks across sev eral frames. F rom experimertal results, w e understand that the design of $m$ depends on the tradeoff betw eenincreasing the detection rate and decreasing the false alarm rate. It has been found that the values of $m$ varies from 2.0 to 4.0 give good results.

Through the above sliding-window thresholding scheme, the detected frames are called potential key frames. F rom experimental results, w eobserved that there are redundant false-alarmed frames, which don't contain significant color information, inside these poten tial key frames. Then, w e adopt a coarse-to-fine strategy to eliminate those false-alarmed frames. In this strategy, these potential key frames is processed one more time by the sliding-window thresholding scheme. After this examination, the detected frames are desired key frames which are then used by the DSQ for the extraction of color palettes. We illustrate the proposed scheme of detecting key frames for compressed video in Figure 2.

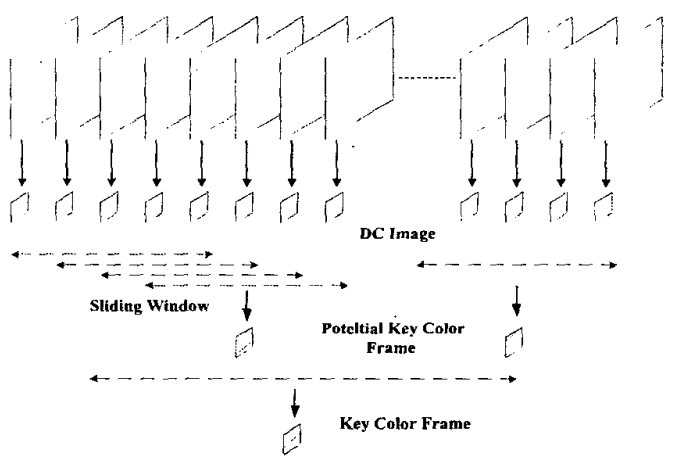

Figure 2: Detection of key frame.

T o do limited-color disply with the color palette extracted by the proposed scheme, w e emplo the same procedures as for the compressed image. Using the w ell-organized boundaries of color cubes partitioned by the DSQ, pixels of each image of decoded MPEG sequence are mapped to their associated representativ e colors.

\section{Shifting-Window Scheme}

If only one fixed color palette is assigned to the whole sequence, the performance of color quantization is getting worse as the sequence length gets longer. On the other hand, if a color palette is designed for every $\mathrm{k} \mathrm{ey}$ frame, a serious visual artifact, screen flicker, may occur when the color palette is changed for these key frames[8]. This problem happens because there is a sudden change of images colors. When the frame buffer of the display con tains animage, a new color palette which belongs to the next image is already active. This phenomenon of screen flicker is sharp and unpleasant to $\mathrm{h}$ uman eyes.

To solve the screen flicker problem, we still use the DSQ to design color palettes for the sequence. But a color palette is designed for each fixed-length shiftingwindow in the sequence. The procedures of color palette design in the video sequence of eac $\mathrm{h}$ window are the same as mentioned above. The key frames in each shifting-window are detected and applied to the DSQ for the color palette design as depicted in Figurel
3. These windows contains o verlapping frames, whik causes the color distribution would not vary too much from window to window even if the color change occurs. As a result, the DSQ can generate smoothly varying color palettes for the sequence if the bit allocation procedure is fixed. In addition, since every en try of the color palette of the processed window w ouldnot differ significantly from that of the next window, screen flic ker is greatly reduced.

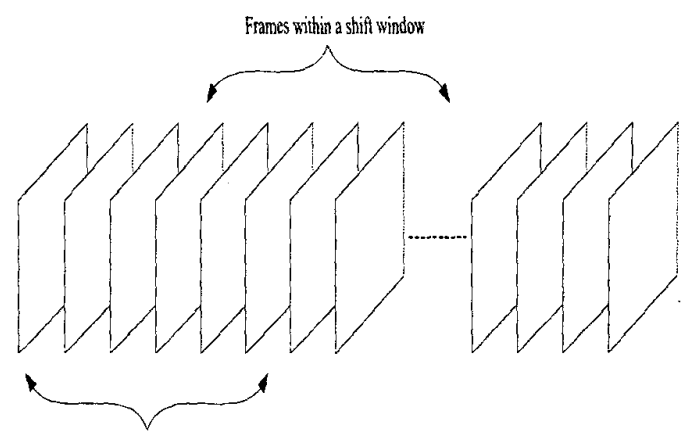

Figure 3: The proposed shifting-window scheme.

\section{Experimental Results and Discussions}

To evaluate the performance of the proposed method for compressed videos, a test sequence "News" which has 300 YUV frames with size $352 \times 240$ is used. The sequence consists of three main video segments which are news conference of a president candidate, a TV news reporter and news of a tropical storm. There are special effects of dissolving, fading in and fading out existed within each transition of tw osegments. This test sequence is first compressed by MPEG-1 compression. Then the proposed scheme is applied to design a color palette of 256 colors on the YUV color space. The color components were quantized in the order of $Y$ (Luminance) first, then $U$ and $V$ last. The numbers of bits in each color component after bit allocation in the DSQ are $4(\mathrm{Y}), 2(\mathrm{U})$ and $2(\mathrm{~V})$.

The detected key frames from the extracted DC sequence are frame $0,3,90,195$ and 210 which are shown in Figure 4. The parameters of criterion 1 and 2 used to find a color change are set to be 7 and 2 , respectively. The sum of hue difference in the first step of scheme of detecting key frames is plotted in Figure 5. As w ecan see, these frames indeed represent significant color difference. Frame 210 is detected because the yellow caption appears. And w eplot in Figure 6 the PSNR distribution of luminance component of the decoded MPEG-1 sequence and the decoded sequence quantized by the proposed scheme. In this figure, the a verage PSNR of the decoded MPEG-1 sequence is $33.9805 \mathrm{~dB}$ and that of the proposed scheme is 32.4647 $\mathrm{dB}$, which shows only about $1.5 \mathrm{~dB}$ lost on average in the color quantization. When we analyze Figure 6 , it is noticed that some peaks or intra-frames among frame 90 and frame 210 have about $5 \mathrm{~dB}$ lost betw een PSNR values of the decoded MPEG- 1 sequence and the proposed scheme. Normally, decoded intra-frames of MPEG-1 sequence are good quality since no motion estimation is involv ed. $F$ or these intra-frames, it shows that the matching of colors of decoded MPEG-1 frame with those of the original frame is better than that of -2R2 representative colors obtained from the proposed 
(a)

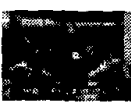

(b)

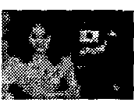

(c)

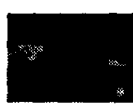

(d)

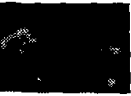

(e)

Figure 4: The DC images of detected key frames: (a)frame 0 (b)frame 3 (c)frame 90 (d)frame 195 (e)frame 210

scheme. How ever since the PSNR values of the proposed scheme for these frames are around $35 \mathrm{~dB}$, w e don't perceive significant degradation of picture quality in experiments. Concerning the computation time, the proposed method took about 8.8 seconds to extract a color palette for the test sequence when using a SUN SPARC20 workstation.

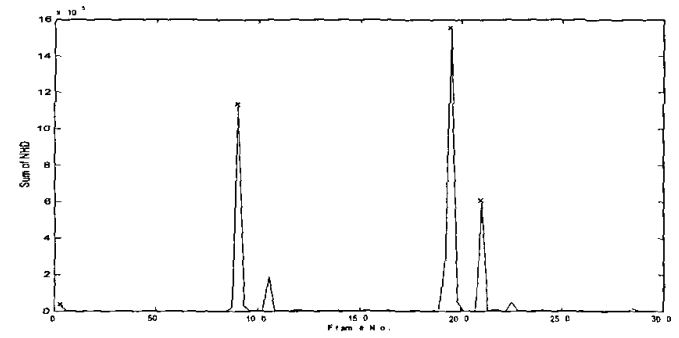

Figure 5: Sum of hue difference plot of "News".
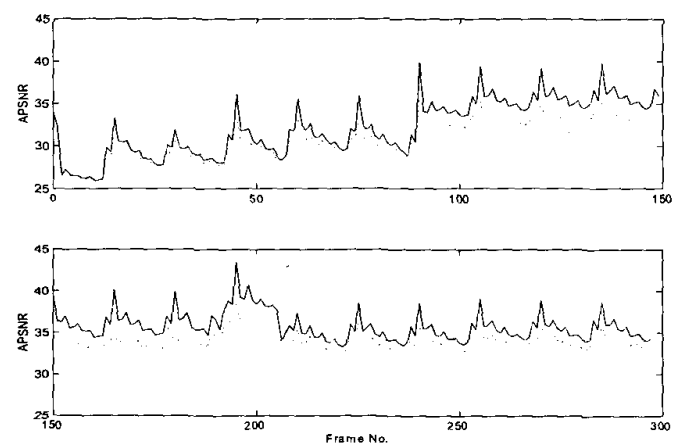

Figure 6: The PSNR in the sequence "News". The solid curv eis for the decoded MPEG-1 sequence and the dotted curve corresponds to the proposed scheme.

We also executed the shifting-window scheme with the size of shifting-window being 150 and the size of overlapping frames betw een neighbouring windows being 75 on the test sequence. This configuration results in three shifting-windows to co ver the test sequence. The designed color palettes for frames $0-149,75-225$ and 150-299 are shown in Figure $7(\mathrm{a})$, (b) and (c). As w ecan see, the color palette of frames 150-299 contains more dark colors which appear in the video clip of the tropical storm. And the gradual changes can be observed among these color palettes. When the corresponding quantized sequence is played badk with frames 0-74 using palette of Figure 15(a), frames 75224 using palette of Figure 15(b), and frames 225-299 using palette of Figure 15(c), we have seen that screen flic ker phenomenon is insipid and acceptable to hman eyes.

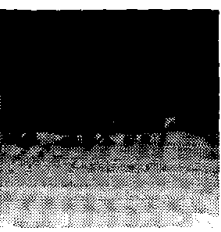

(a)

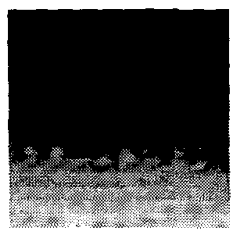

(b)

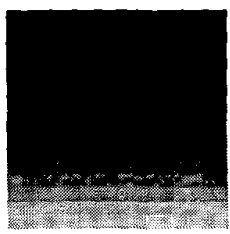

(c)
Figure 7: The color palettes for the sequence "News" when the shifting-windows scheme is employed.

\section{References}

[1] P. Hekbert, "Color image quantization for frame buffer display", Comput. Graph., vol. 16, no. 3, pp. 297-397, Jul. 1982.

[2] S. C. P ei and C. M. Cheng, "Dependent scalar quantization of Color Images", IEEE T rans. Circuits and Systems for Video Technology, vol. 5, no. 2, pp. 124-139, Apr.1995.

[3] "MPEG Video Committee Draft," ISOIEC/JTC1/SC29/WE11/MPEG 90/176, Dec. 1990.

[4] R. Gray, "Vector quantization", IEEE ASSP Mag., vol. 1, pp. 4-29, Apr. 1984.

[5] B. L. Y eo andB. Liu, "Rapid Scene Analysis on Compressed Videos", IEEE T ans. Cir cuits and Systems for Video T echnolgy, vol. 5, no, 6, pp. 533-544, Dec. 1995.

[6] Y. Nakajima, "a Video Browsing Using Fast Scene Cut Detection for an Efficient Net w ork edideo Database Access", IEICE T ans. Inf. and Syst., vol.E77-D, no. 12, pp. 1355-1364, Dec. 1994.

[7] B. L. Yeo and B. Liu, "On the extraction of DC sequences from MPEG compressed video", International Conferenc eon Image Proessing, vol. 2 pp. 260-263, Oct. 1995.

[8] E. Roytman and C. Gotsman, "Dynamic color quantization of video sequences", IEEE Trans. Vi sualization and Comperter aphics, vol. 1, no. 3, pp. 274-286, Sep. 1995. 